\title{
Molecular characterisation, evolution and expression analysis of g-type lysozymes in Ciona intestinalis
}

\author{
Felicia Di Falco, Matteo Cammarata, Aiti Vizzini* \\ Marine Immunobiology Laboratory, Department of Biological, Chemical, Pharmaceutical Science and Technology, University of Palermo, Via Archirafi 18,
} Palermo, Italy

\section{A R T I C L E I N F O}

\section{Article history:}

Received 26 July 2016

Received in revised form

18 September 2016

Accepted 19 September 2016

Available online 20 September 2016

\section{Keywords:}

Ascidian

Lysozymes g-type

Inflammation

LPS

Ciona intestinalis

\begin{abstract}
A B S T R A C T
Lysozyme is an important defense molecule of the innate immune system. Known for its bactericidal properties, lysozyme catalyzes the hydrolysis of b-(1,4)-glycosidic bonds between the N-acetyl glucosamine and $\mathrm{N}$-acetyl muramic acid in the peptidoglycan layer of bacterial cell walls. In this study, the complete coding sequence of four g-type lysozymes were identified in Ciona intestinalis. Phylogenetic analysis and modelling supported the hypothesis of a close relationship with the vertebrate g-type lysozymes suggesting that the $C$. intestinalis g-type lysozyme genes (CiLys-g1, Cilys-g2, CiLys-g3, CiLys-g4) share a common ancestor in the chordate lineage. Protein motif searches indicated that $C$. intestinalis gtype lysozymes contain a GEWL domain with a GXXQ signature, typical of goose lysozymes. Quantitative Real-Time PCR analysis results showed that transcripts are expressed in various tissues from C. intestinalis. In order to determine the involvement of $C$. intestinalis g-type lysozymes in immunity, their expression was analyzed in the pharynx, showing that transcripts were significantly up-regulated in response to a challenge with lipopolysaccharide (LPS). These data support the view that CiLys g-type are molecules with potential for immune defense system against bacterial infection.
\end{abstract}

๑) 2016 Elsevier Ltd. All rights reserved.

\section{Introduction}

Lysozyme is a ubiquitous bacteriolytic enzyme produced by diverse groups of organisms, ranging from bacteria and bacteriophages to fungi, plants and animals (Jollès and Jollès, 1984). It catalyzes the hydrolysis of 1, 4-beta-linkages between N-acetyl- $d$ glucosamine (NAG) and N-acetylmuramic acid (NAM) in peptidoglycan heteropolymers of prokaryotic cell walls, leading to the breakdown of bacterial cells (Smirnow and Wislowska, 2001; Nilsen et al., 1999). As a result, lysozyme acts directly on Grampositive bacteria, causing the lysis of their outermost peptidoglycan layer. Gram-negative bacteria, however, are not directly damaged by lysozyme as their outer membrane is significantly coated with lipopolysaccharide (LPS) moieties. Instead, the outer membranes of Gram-negative bacteria must first be disrupted by cationic antimicrobial peptides that expose the inner peptidoglycan layer of bacteria to lysozyme (Banks et al., 1986; Hancock and Scott, 2000; Ibrahim et al., 2002).

Based on differences in structural, catalytic and immunological characteristics, lysozymes are generally classified into six main

\footnotetext{
* Corresponding author.

E-mail address: aiti.vizzini@unipa.it (A. Vizzini).
}

types: chicken (c-type) (Hultmark, 1996), goose (g-type) (Prager and Jollès, 1996), invertebrate (i-type) (Jollès and Jollès, 1975), T4 phage (phage-type) (Fastrez, 1996), bacterial (Holtje, 1996), and plant (Beintema and Terwisscha van Scheltinga, 1996). The g-type lysozyme was initially identified as an antibacterial enzyme in egg whites from the Embden goose (Canfield and McMurry, 1967), but it was later found to exist in the egg whites of several other bird species (Prager et al., 1974). More recently, the g-type lysozyme has been identified in vertebrate species, including mammals (Nakano and Graf, 1991), fish (Mohanty and Sahoo, 2010), urochordates (Nilsen et al., 2003) and molluscs (Zhao et al., 2007; Zhang et al., 2012).

Lysozyme is a well-known antibacterial protein which is active against Gram-positive bacteria such as Staphylococcus aureus, Micrococcus luteus, Bacillus stearothermophilus and Clostridium tyrobutyricum (Proctor and Cunningham, 1988). It is also known to act as an opsonin and as an activator of the complement system and circulating phagocytes (Jollès and Jollès, 1984; Grinde, 1989). In addition to these antibacterial functions, some lysozyme family members have been demonstrated to have antiviral (Ferrari et al., 1959; Lee-Huang et al., 1999), anti-inflammatory activities (Jollès and Jollès, 1984; Samarayanake et al., 1997; Ogundele, 1998; 
Zhang et al., 2008), to be involved in immune modulatory (Valisena et al., 1996; Rymuszka et al., 2005) and antitumor activities (Sava et al., 1989), thus, it is possible that lysozymes function as multipurpose defense factors.

Ascidians (subphylum: Tunicata) occupy a key phylogenetic position in chordate evolution and are considered the sister group of vertebrates (Zeng and Swalla, 2005; Delsuc et al., 2006; Tsagkogeorga et al., 2009). They are proto-chordates which possess an innate immune system, including inflammatory humoral and cellular responses. An inflammatory response induced by LPS injection in the body wall of $C$. intestinalis is a well-established model for the analysis of regulator and effector inducible host defense molecules of the innate immune system (Bonura et al., 2009; Parrinello et al., 2008, 2010; Vizzini et al., 2012, 2013; 2015a, 2015b; 2016). In the present paper, we report on the identification, characterization and expression of $C$. intestinalis g-type lysozymes. Phylogenetic analysis was conducted to determine their evolutionary relationships. Real-Time PCR analysis revealed that CiLys$\mathrm{g}(1-4)$ are expressed in several type of tissue, and their transcription is up-regulated by LPS inoculation.

\section{Materials and methods}

\subsection{Tunicates and LPS inoculation}

Ascidians were collected from Sciacca Harbour (Sicily, Italy), maintained in tanks with aerated sea water at $15^{\circ} \mathrm{C}$, and fed every second day with a marine invertebrate diet of coraliquid (Sera Heinsberg, Germany). LPS (Escherichia coli 055:B5, LPS, SigmaAldrich, Germany) solution was prepared in sterile sea water (12 $\mathrm{mM} \mathrm{CaCl}_{2}, 11 \mathrm{mM} \mathrm{KCl}, 26 \mathrm{mM} \mathrm{MgCl}_{2}, 43 \mathrm{mM}$ Tris $\mathrm{HCl}, 0.4 \mathrm{M}$ $\mathrm{NaCl}, \mathrm{pH}$ 8.0). LPS solution (100 $\mu \mathrm{g}$ LPS in $100 \mu \mathrm{l}$ sea water per animal) was inoculated into the tunic matrix close to the pharynx wall at the median body region. Ascidians, both untreated (naive ascidians) and injected with MS (sham ascidians), were used as controls.

\subsection{Total RNA extraction}

Ascidian tissue fragments ( $200 \mathrm{mg}$ ) explanted at various times (from 1 to $72 \mathrm{~h}$ ) were immediately soaked in RNAlater Tissue collection solution (Ambion, Austin, TX), and stored at $-80^{\circ} \mathrm{C}$. Total RNA extraction was performed by using an RNAqueousTM-Midi Kit purification system (Ambion, Austin, TX).

\subsection{Cloning and sequence analysis}

A search conducted in the Ensembl genome browser identified the sequence: CiLys-g1 (ENSCING00000007365), CiLys-g2 (ENSCING00000024417), Cilys-g3 (ENSCING00000018223), CiLys-g4 (ENSCING00000007365). The sequence of the cDNA was obtained by using the GeneRacerTM kit (Invitrogen, USA). 5'-and 3' RACE was conducted using the primers listed in Table 1. The overlapping RACE products were cloned into the $\mathrm{pCR}^{\mathrm{TM}}$ IIvector (TA Cloning Kit, Invitrogen) and sequenced. They contained the complete coding regions.

\subsection{Bioinformatic analysis}

The full length CiLys-g(1-4) cDNA were analyzed using the ExPASy translation tool (http://web.expasy.org/translate/) to obtain their ORF region, leader and trailer sequences (UTR), and the nucleotide sequence was translated into a protein sequence. A BLAST (http://blast.ncbi.nlm.nih.gov/Blast) search was conducted to identify the known protein sequences that are homologous to
Table 1

Primers used for cloning and expression.

\begin{tabular}{|c|c|c|}
\hline Gene & Primer sequence $\left(5^{\prime}-3^{\prime}\right)$ & Application \\
\hline \multirow[t]{4}{*}{ CiLys-g1 } & 5'-TAACCCATACCCATGCCCGT-3' & RACE5' \\
\hline & 5'-GCTTGCCCAATCTCGTCTTT-3' & NESTED5' \\
\hline & 5'-TGACGGGGATTCGAACAGGA-3' & RACE3' $^{\prime}$ \\
\hline & 5'-ATCTGGAAAGTGTGGAGGGC-3' & NESTED3' \\
\hline \multirow[t]{4}{*}{ CiLys-g2 } & $5^{\prime}$-CTCCCAAACCCTTGCTGTTC-3' & RACE5' \\
\hline & 5'-AATTAGATCGGGCGGCCTTA-3' & NESTED5' \\
\hline & 5'-GCAATGGCGACTCGTTTCAA-3' & RACE3' \\
\hline & 5'-GCTGAACGAGAATGGCTACG-3' & NESTED3' \\
\hline \multirow[t]{4}{*}{ CiLys-g3 } & 5'-TGCGACGACTCTAACACTTG-3' & RACE5' \\
\hline & 5'-CGTACATTGCTGACCCCAAA-3' & NESTED5' \\
\hline & 5'-AAAAGATCTGCGTGCGATGG-3' & RACE3' $^{\prime}$ \\
\hline & 5'-AGGTTGATAAGCGGCACCAT-3' & NESTED3' \\
\hline \multirow[t]{4}{*}{ CiLys-g4 } & $5^{\prime}$-TCCTGCCACCATTTTACCAC-3' & RACE5' \\
\hline & 5'-AGCCACTACATCGCTAGAGT-3' & NESTED5' \\
\hline & 5'-TTGCTTTTCCTGTTCGTCGG-3' & RACE3' \\
\hline & 5'-GACGATCGTTACCACACCAT-3' & NESTED $3^{\prime}$ \\
\hline \multirow[t]{2}{*}{ CiLys-g1 } & 5'-AACTTTGTATGGACGCTGCTG-3' & Real-time PCR \\
\hline & $5^{\prime}$-GCCCCTGCACGACTTTCA-3' & Real-time PCR \\
\hline \multirow[t]{2}{*}{ CiLys-g2 } & $5^{\prime}$-CACGGTGCCGACACAAAGT-3' & Real-time PCR \\
\hline & 5'-GCGCCTTGTAAAATGTGATCTC-3' & Real-time PCR \\
\hline \multirow[t]{2}{*}{ CiLys-g3 } & $5^{\prime}$-GCAAGCCGCGAAAGCA-3' & Real-time PCR \\
\hline & $5^{\prime}$-TCACCAAGCCCGTCTTTGTC- $3^{\prime}$ & Real-time PCR \\
\hline \multirow[t]{2}{*}{ CiLys-g4 } & 5'-CGGCGTAGCCATCGCTTA-3' & Real-time PCR \\
\hline & $5^{\prime}$-CGGTGGTGTCAGTGTTGTAGAT-3' & Real-time PCR \\
\hline \multirow[t]{2}{*}{ Actin } & 5'-TGATGTTGCCGCACTCGTA-3' & Real-time PCR \\
\hline & 5'-TCGACAATGGATCCGGT-3' & Real-time PCR \\
\hline
\end{tabular}

CiLys-g(1-4). The Exon-Intron Graphic Maker (http://wormweb. org/exonintron) was used to create an image of the genomic organization of CiLys-g genes and for comparison. Physical and chemical parameters such as molecular mass, and theoretical isoelectric point were computed using the Prot-Param tool on ExPASy (http://www.expasy.org/tool/protparam/). The NCBI Conserved Domain database (http://www.ncbi.nlm.nih.gov/Structure/cdd/ wrpsb.cgi) was used to predict domain architecture and other conserved domains based on sequence homology. Functional motifs were determined by comparison on the Prosite database (http://prosite.expasy.org/scanprosite/). The putative cleavage site of the signal peptide was predicted by SignalP 4.0 (http://www.cbs. dtu.dk/services/SignalP/). The subcellular location of lysozyme proteins was predicted using the MultiLoc tool (http://abi.inf.unituebingen.de/Services/MultiLoc/). Multiple sequence alignment was conducted using CLC (Version 7.0.0). A secondary structure was evaluated using Polyview (http://polyview.cchmc.org). Different 3D structures were predicted using the I-TASSER program (http:// zhanglab.ccmb.med.umich.edu/I-TASSER) and the structures were validated by Ramachandran plot analysis (http://mordred.bioc.cam. ac.uk/ rapper/rampage.php) to obtain the best structure among those predicted. Finally, a phylogenetic tree using Neighbor-joining method was constructed using MEGA 6.0 after 1000 bootstrap iterations.

\subsection{Real-Time PCR analysis}

Tissue Differential expression of the CiLys-g(1-4) cDNAs was studied by Real-Time PCR using the Sybr-Green method and the specific sets of primers listed in Table 1. Real-Time PCR analysis was performed using the Applied Biosystems 7500 Real-Time PCR System. Tissue Differential expression was performed in a $25 \mu \mathrm{lPCR}$ reaction containing $2 \mu \mathrm{l}$ cDNA converted from 250 ng of total RNA, $300 \mathrm{nM}$ forward and reverse primers, and $12.5 \mu \mathrm{l}$ of Power SybrGreen PCRMasterMix (Applied Biosystems).

The 50 cycles of the two-step PCR program consisted of initial polymerase activation for $3 \mathrm{~min}$ at $95^{\circ} \mathrm{C}$, followed by a denaturing step at $95^{\circ} \mathrm{C}$ for $15 \mathrm{~s}$, and then annealing/extension was carried out 
at $60{ }^{\circ} \mathrm{C}$ for $45 \mathrm{~s}$ when the fluorescent signal was detected. Each set of samples was run three times and each plate contained quadruplicate cDNA samples and negative controls.

The amplification specificity was tested using Real-Time PCR melting analysis. To obtain sample quantification, the $2^{-\Delta \Delta \mathrm{Ct}}$ method was used and the relative changes in gene expression were analysed as described in the Applied Biosystems Use Bulletin N.2 $(\mathrm{P} / \mathrm{N}$ 4303859). The amount of CiLys-g(1-4) transcripts from different tissues was normalised to actin in order to compensate for variations in input RNA amounts. Relative CiLys-g(1-4) expression was determined by dividing the normalised value of the target gene in each tissue by the normalized value obtained from the untreated tissue.

\subsection{Statistical methods}

Multiple comparisons were performed using one-way analysis of variance (ANOVA), and different groups were compared by using Tukey's $t$-test. Standard deviations were calculated on four experiments. $\mathrm{P}<0.05$ was considered statistically significant.

\section{Results}

\subsection{Sequence analysis of g-type lysozymes in C. intestinalis}

A search in the Ensembl genome browser identified only g-type lysozymes: CiLys-g1 (ENSCING00000007365), CiLys-g2 (ENSCING00000024417), CiLys-g3 (ENSCING00000018223), Cilys-g4 (ENSCING00000007365)

The full-length mRNA of g-type lysozymes was isolated using a $5^{\prime}$ and $3^{\prime}$ RACE strategy. The cDNA and amino acid sequence analysis showed that: CiLys-g1(GenBank Accession No. KX 761980) presents a 5'-UTR of $80 \mathrm{bp}$, an ORF of $906 \mathrm{bp}$ and a 3'-UTR of $84 \mathrm{bp}$, encoding 301 amino acids with a predicted molecular size of $39.3 \mathrm{kDa}$ and a pI of 6.93 (Fig. 1); CiLys-g2 (KX761981) presents a 5'UTR of $41 \mathrm{bp}$, an ORF of $564 \mathrm{bp}$, and a 3'-UTR of 98, encoding 186 amino acids with a predicted molecular size of $26.4 \mathrm{kDa}$ and a pI of 7.8 (Fig. 1); CiLys-g3 (KX761982) presents a 5'-UTR of 59 bp, an ORF of $456 \mathrm{bp}$ and a 3'-UTR of $197 \mathrm{bp}$, encoding 151 amino acids with a predicted molecular size of $27.7 \mathrm{kDa}$ and a pI of 9.79 (Fig. 1); CiLysg4 (KX761983) presents a 5'-UTR of $58 \mathrm{bp}$, an ORF of $609 \mathrm{bp}$ and a 3'-UTR of $91 \mathrm{bp}$, encoding 202 amino acids, predicted molecular size of $28.5 \mathrm{kDa}$ and a pI of 7.76 (Fig. 1). In silico analysis, using Signal P 4.1 and the DELTA BLAST program to evaluate the presence of conserved domains, showed that: CiLys-g1 presents a predicted signal peptide of 16 amino acids and a Lytic transglycosylase-like (SLT) domain (51-162); CiLys-g2 presents a signal peptide of 20 amino acids and a Lysozyme-like domain (2-174); CiLys-g3 does not present a signal peptide and shows a Lytic transglycosylase-like (SLT) domain (19-134);CiLys-g4 presents a signal peptide of 16 amino acids and a Lytic transglycosylase-like 2 (SLT2) domain (26-101) (Fig. 1). The MultiLoc tool was used to predict the subcellular location of lysozyme proteins: CiLys-g1, CiLys-g3, CiLys-g4 showed extracellular lysozymes, while CiLys-g2 showed an intracellular lysozyme. The deduced amino acid sequences of C. intestinalis g-type lysozymes, examined in GeneBank through BLAST analysis, showed significant homologies with components of the g-type lysozymes family: CiLys-g1 has an identity of 56\% and a positivity of $67 \%$ with the Lysozyme g-like 1 of Danio rerio (NP 001002706.1 ); an identity of $39 \%$ and a positivity of $57 \%$ with the Lysozyme g 2 precursor of Homo sapiens (NP_783862.2); CiLys-g2 has an identity of $31 \%$ and a positivity of $54 \%$ with the Lysozyme $g$ of $D$. rerio (NP 001002706.1 ); an identity of $26 \%$ and a positivity of $51 \%$ with the Lysozyme $g$ precursor of $H$. sapiens (NP_783862.2); CiLys-g3 has an identity of $51 \%$ and a positivity of $68 \%$ with the
Lysozyme g 1 of $D$. rerio (NP 001002706.1); an identity of 37\% and a positivity of $60 \%$ with the Lysozyme g 2 precursor of $H$. sapiens (NP_783862.2); CiLys-g4 has an identity of 55\% and a positivity of $68 \%$ with the Lysozyme $g 1$ of $D$. rerio (NP 001002706.1); an identity of $35 \%$ and a positivity of $53 \%$ with the Lysozyme $g$ precursor of H. sapiens (NP_783862.2).

The genomic organization of the CiLys-g genes was determined and compared with those of human and $D$. rerio lysozyme type-g genes (Supplementary data, Fig. S1). The CiLys-g1 and CiLys-g4 genes contained five exons and four introns like the $D$. rerio type $g$ lysozyme gene, but unlike the CiLys-g2 and CiLys-g3 genes that contained 3 exons and 2 introns, whereas the human g-type lysozyme 1 gene contained eight exons and seven introns, and lysozyme g-type 2 contained six exons and five introns, with an increase in the number of exons in the human lysozyme genes compared to the lysozyme genes of $C$. intestinalis.

\subsection{Alignment and structural analysis}

BLAST analysis with DELTA-BLAST program showed that the CiLys-g proteins have a GEWL domain, which belongs to a lysozyme-like superfamily, and a GXXQ motif, which is a signature of goose family lysozyme. In Fig. S2, by using the CLC workbench 6.4 alignments, amino acids potentially important for lysozyme catalytic activity (Glu, Asp, Asp) and for binding to ligands (Gly) were found conserved, and a Lytic transglycosylase-like (SLT) domain was also identified.

Secondary structure analysis using the Polyview online tool showed an $\alpha$ helix region but no $\beta$-sheet region in the Cilys-g proteins. Five distinct $\alpha$-helices were found in the secondary structure, which is a conserved feature among the g-type lysozymes. Tertiary structure analysis in PyMol revealed that the 3D structures of CiLys-g proteins, which was predicted using the ITasser online server, also showed five helix regions (Fig. 1). The active residues of the NAG binding site were distributed in both the coil and helix regions. The conserved GXXQ motif and the conserved catalytic residues (Glu) and (Asp) were found to be located nearer to the hydrophobic core region.

Fig. 1 A-B shows the CiLys-g1 molecular model resulting from the super-imposition of the 117-301 residue sequence, which corresponds to the mature peptide and shares 55.43\% identity with the template. The homology modelling process was performed on the basis of the known crystal structure of Australian black swan egg white lysozyme (1gbs.1.A). The amino acids involved in binding to the substrate $\left(\mathrm{Gly}^{118}, \mathrm{Tyr}^{263}, \mathrm{Gly}^{266}\right)$ and the catalytic site $\left(\mathrm{Glu}^{188}\right.$, $\mathrm{Asp}^{213}$ ) were found to be conserved.

Fig. 1 C-D shows the CiLys-g2 molecular model resulting from the super-imposition of the 17-173 residue sequence, which corresponds to the mature peptide and shares $32.9 \%$ identity with the template. The homology modelling process was performed on the basis of the known crystal structure of Anser anser goose lysozyme (1531.1.A). The amino acids involved in binding to the substrate $\left(\operatorname{Tyr}^{135}, \mathrm{Gly}^{138}\right)$ and the catalytic site $\left(\mathrm{Arg}^{60}, \mathrm{Gln}^{82}, \mathrm{Gln}^{103}\right)$ were found to be conserved.

Fig. 1 E-F shows the CiLys-g3 molecular model resulting from the super-imposition of the 4-151 residue sequence, which corresponds to the mature peptide and shares $51.70 \%$ identity with the template. The homology modelling process was performed on the basis of the known crystal structure of Struthio camelus lysozyme $g$ (3mgw.1.A). The amino acids involved in binding to the substrate $\left(\mathrm{Tyr}^{113}, \mathrm{Gly}^{116}\right)$ and the catalytic site $\left(\mathrm{Glu}^{38}, \mathrm{Asp}^{48}, \mathrm{Asp}^{63}\right)$ were found to be conserved.

Fig. $1 \mathrm{G}-\mathrm{H}$ show the CiLys-g4 molecular model resulting from the super-imposition of the 18-202 residue sequence, which corresponds to the mature peptide and shares $54.35 \%$ identity with the 


\section{CiLys-g1} TAGAGGAACTAACTGTTCAAGATGAAGCAATTGTTGGTTATCTGCCTTTTGATTGAAATA

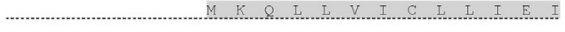

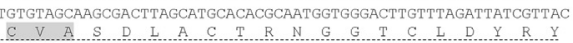
TACTTATGCACTGCTGGTTATGAACAAGGATTATGTGACGGGGATTCGAACAGGAAGTGC

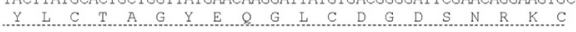
TGCCAGGAATGCGATAATACTTGCATCTCAAACGAGAATAGTTATGCTTCATGCTGTGAC C $S$ E C D N T C I S N E N S Y A S C C D

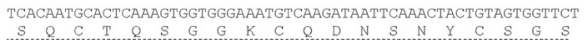
TATTCATCTGGAAAGTGTGGAGGGCCTT

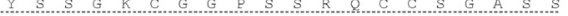
$G$ G A E G C Y G N I Y N T D T T G A S C ACCACGTCGAGTCAAGATAACTTAGGATATTGCGGCATATCAGGGTCTCGTCAATTGGCO

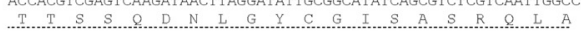
GCTACA GATCTT AACAGGATGTCCCAATACAAAGACGAGATT GAACAAGCCGGTTACCAA A.P D I N R M S O Y K D E I E O A G Y O CTTTGTATGGACGCTGCTGTTATTGCAGGTATCATCAGTCGTGAAAGTCGTGCAGGGGCA I $C M D A A-A G I A S E S R A G$

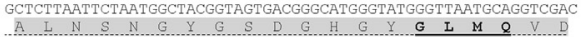
GATCGTTACCACACCATACAAGGCGACCCT ACATCTCT TGACCATATATT ACAAGGCACT D R Y H T I O G D P T S L D H I L O G $\mathrm{T}$ GGCATCCTTATATCTAATATTGGACAAATATCTTCGAAATTTCCACCTTGGGACCAAAAC G T L T S N T G $Q$ T S S K F P P W D $Q N$ ATGGACTTAAAAGGCGGCATATGCGCTTATAATATAGGTGTTGGGGCGTGTGGTCTTAT $M$ D L K G G I C C A Y GATAACATGGACGTCGGTACAACTGGGGATGACTACTOTIACC. D N M D Y G T F G D D Y S S D V V A R CAGTATTATAAAGACAACGGGTATTAGGGAATGGATTATAAGCTATGATGTAATAGGTGG Q Y Y K D N $\quad$ Y Y * *

1021 TAAAATGGTGGCAGGATATT GGCGATTCTGGTAATAAATACAACCTATATAAAAAAARAA
1081 AAAAAAAAAAAAA
A

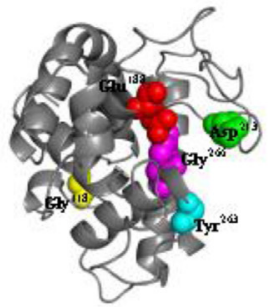

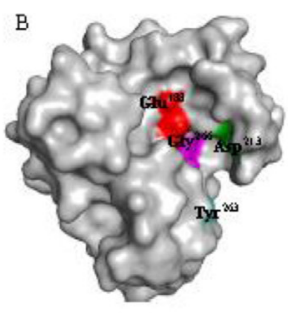

CiLys-g2

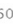

120

180 240

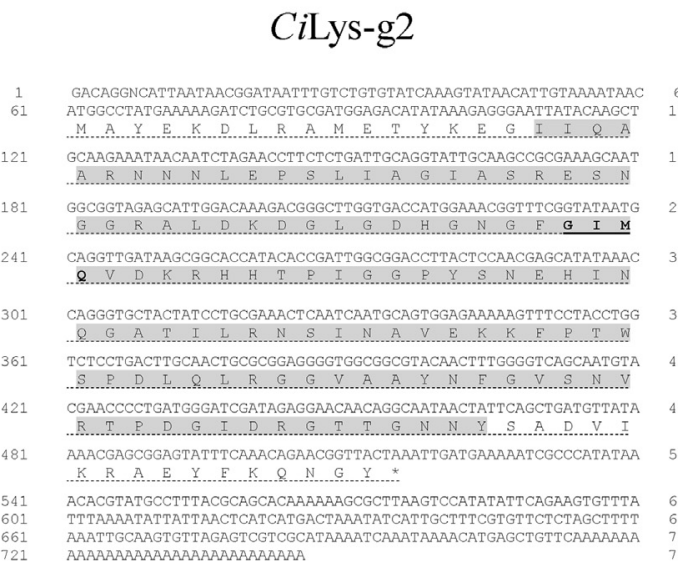

C

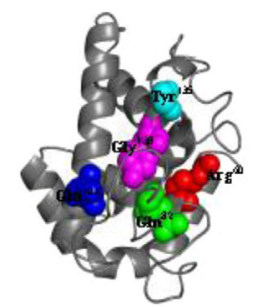

D

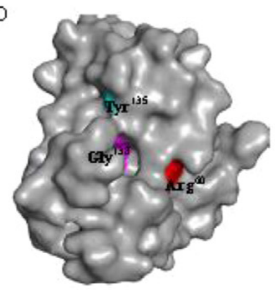

CiLys-g3

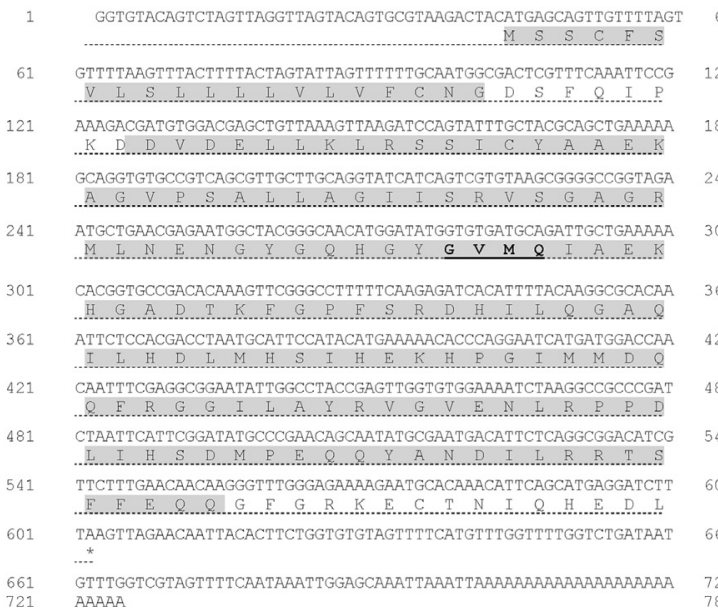

60

120

180

240

300

360

420

480

540

600

660

720

E

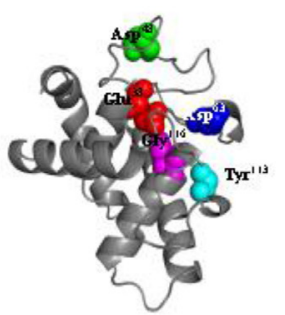

F

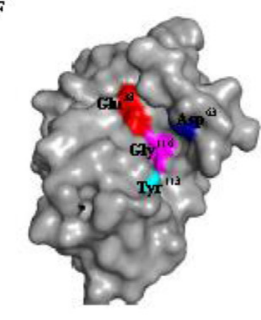

\section{CiLys-g4}

1 AATCGCTGAAACCAAGACCGT TATCACAACTCGCGAGTTGAACGAATACGGTTTGAAA ATGTTGAAGGTATTTTTGCTTTTCCTGTTCGTCGGCGTAGCCATCGCTTATACCGGTTGC Th N I Y N T D T T G C S S D D N I G Y S G AACTTGGGTTACAGCGGAATCTCCGCT TCGAGGCAAATGGCCGCAACAGATCTGTCCAGA

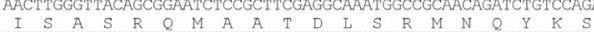
ATGAACCAATACAAAAGTAACATTGAGAATGCTGGACGTCAACTGTGTATGGATCCITCT N I E N A G R Q L C M D P S V I A G I I I GTCATTGCAGGCATCATTAGCAGGGAACTCGTGCGGGGCTACCCTTGGTTCAGACGGT TACGSCGCAGATGGACACGGA CAY CAAGGTGGCCCTACATCTCTTGACCATATAT TACAAGGCACTGGCATCCTTATATCAAAT 480 ATTGGACAAATATCTTCGAAATT TCCATCT TGGGACCAAAACATGGACTTAAAAGGCGGC 540 K F P S W D O N M D L K G G I C A Y N I ATATGCGCTTATAATATAGGTGTTGGGGCGTGTGGTCTTATGATAGCATGGACGTCGGT 600 ACAACTGGGGATGACIACTCTAGCGATGTAGTGGCTAGAGCCCAGTATTATAAAGACAAC 660 (5) GGGTATTAGGGAAATGGGATTATAAGCTATGATGTAATAGGTGGTAAAATGGTGGCAGGA 720 G. $\mathrm{T}$ *

721 TATTGGCGATTCTGGTAATAAATACAACCTATATAATT TAAAAAAAAAAAAAAAAAAAAA

G

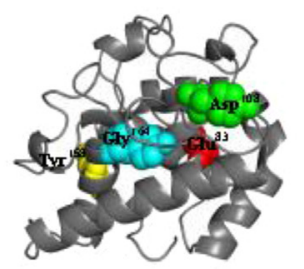

H

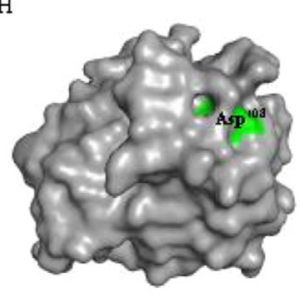

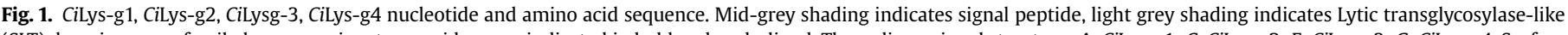

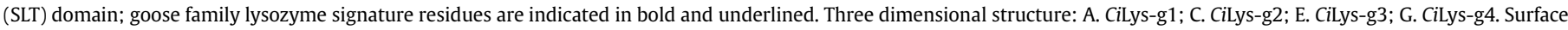
view: B. CiLys-g1; D. Cilys-g2; F. CiLys-g3; H. CiLys-g4. The active residues are highlighted as coloured spheres, with numbers representing the location. 
template. The homology modelling process was performed on the basis of the known crystal structure of Struthio camelus lysozyme $g$ (3mgw.1.A). The amino acids involved in binding to the substrate $\left(\mathrm{Tyr}^{158}, \mathrm{Gly}^{164}\right)$ and the catalytic site $\left(\mathrm{Glu}^{83}, \mathrm{Asp}^{108}\right)$ were found to be conserved.

\subsection{Phylogenetic analysis}

By using the MEGA 6 program, CiLys-g proteins were aligned with vertebrate and invertebrate members of lysozyme superfamily proteins and a phylogenetic tree was constructed using the Neighbor-joining method. The tree shows three main clusters (Fig. S3). The first cluster includes, vertebrate and invertebrate gtype lysozymes as well as $C$. intestinalis g-type lysozymes; the second cluster includes invertebrate i-type lysozymes; the third cluster includes vertebrate and invertebrate c-type lysozymes. This analysis suggests that g-type lysozymes share a common ancestor and that the i-type and c-type may be closely interrelated (Fig. S3).

\subsection{Differential gene expression of g-type lysozymes in different tissues}

The spatial expression pattern of g-type lysozyme mRNA in adult $C$. intestinalis was investigate by quantitative Real-Time PCR analysis of total RNA samples from different tissue (pharynx, ovary, stomach, intestine). Expression was detected in all tissues. As shown in Fig. 2 Cilys-g1 expression was higher in stomach and intestine, and lower in ovary and pharynx tissue; CiLys-g2 expression was higher in pharynx and intestine, and lower in ovary and stomach tissue; CiLys-g3 expression was higher in intestine, and lower in ovary, stomach and pharynx tissue; CiLys-g4 expression was higher in stomach and lower in intestine, ovary and pharynx tissue.

\subsection{G-type lysozyme genes expression is upregulated by LPS}

To study the involvement of the g-type lysozymes of C. intestinalis in inflammatory response induced after LPS challenge, the expression pattern of CiLys-g(1-4) mRNAs in naive, sham and LPS-challenged ascidians was examined by Real-Time PCR analysis.

RealTime PCR analysis of the inflamed ascidian pharynx showed enhanced g-type lysozymes mRNA levels as an effect of the LPS challenge (Fig. 2). To examine the temporal course of the response, four ascidians in three distinct experiments were examined at increasing post-inoculation time points $(1,2,4,8,12,24,48,72 \mathrm{~h})$. At each time point, four sham ascidians were the controls (Fig. 2). CiLys-g1and CiLys-g2 gene expression was significantly boosted at 24-72 h, CiLys-g3 gene expression was significantly boosted at $24 \mathrm{~h}$ and $72 \mathrm{~h}$ and CiLys-g4 gene expression was significantly boosted at $1 \mathrm{~h}$ and $2-4 \mathrm{~h}$, and increased at 8-48 $\mathrm{h}$. The response by sham ascidians indicates that the inoculation procedure did not significantly modulate mRNA expression (Fig. 2).

\section{Discussion}

The immune system is an important physiological mechanism that protects the organism against invading pathogens. Lysozyme has been characterised as an important protein of the innate immune response, and has been evolutionarily conserved throughout invertebrates and vertebrates. The archetypal lysozyme, which has served as a model for studies on enzyme structure and function is the c-type. Analysis of available genome sequences of $C$. intestinalis reveals only g-type homologue lysozyme genes. The goose egg lysozyme (Simpson and Morgan, 1983) was found to be a secreted protein with a signal peptide. G-type lysozyme genes in birds, with the exception of the chicken lysozyme g2, and mammal contain predicted signal sequences for protein secretion (Nile et al., 2004). In contrast most fish g-type lysozymes do not have the signal peptide at their $\mathrm{N}$-terminal sequence, suggesting that they are not secreted from cells (Irwin and Gong, 2003; Kyomuhendo et al., 2007).

In this study, we identified and characterised the mRNA of the four C. intestinalis CiLys-g proteinsthat encoded for tree extracellular lysozymes (CiLys-g1, CiLys-g3,CiLys-g4), and one intracellular lysozyme (CiLys-g2). G-type lysozymes are basic proteins and, in general, present a high isoelectric point $(\mathrm{pI})$. We observed that the g-type lysozymes of $C$. intestinalis cover a wide range of $\mathrm{pI}$ values (from 6.93 to 9.79), suggesting a specialisation for specific tissue or for their intracellular or extracellular location.

Analysis of the alignment and structure of the CiLys-g proteins revealed that the mature proteins have a GEWL domain which is specific for the lysozyme g-type and a GXXQ signature which is specific for the Goose Lysozyme. In particular, in CiLys-g3 all three catalytic residues (Glu, Asp, Asp) responsible for the binding of the lysozyme to the bacterial cell wall turned out to be highly conserved (Fu et al., 2013) whereas in CiLys-g1 and CiLys-g4, only two catalytic residues (Glu, Asp) were found to be conserved. Structure and surface analysis revealed that the conserved GXXQ motif among the Cilys-g lysozymes is located nearer to the hydrophobic core region. This suggests that the GXXQ motif is involved in the process of binding to the bacterial NAM-NAG and to the processing of pathogens. Multiple sequence alignments revealed that glycine residues remained conserved among the sequences, including fish, birds and humans and $C$. intestinalis. These findings indicate the importance of glycine residues in the structural conservation of g-type lysozymes. Thunnissen et al. (1995) confirmed, using a mutation study in which the mutant without glycine residue showed a declined activity, that the glycine residue is essential for lysozymal activity. Phylogenetic analysis of vertebrate and invertebrate g-type, i-type, c-type lysozymes supported the idea of their evolution from a common lysozyme ancestral gene and the conclusion that the i-type and c-type are more closely associated that the g-type. Genomic comparison among the g-type lysozymes of $D$. rerio, humans and $C$. intestinalis showed that the number of exons varies, ranging from only three for the CiLys-g2 and -g3 of ascidians to height in human lysozyme g1, and that the structural similarities of g-type lysozymes are not reflected by their genomic organization, since the exon-intron pattern of their genes is very different.

The spatial expression pattern of the mRNA of g-type lysozymes in adult $C$. intestinalis was investigated for different tissues (pharynx, ovary, stomach, intestine) and was detected in all tissues, but the level of mRNA was highest in the pharynx, stomach and intestine, which is consistent with suggestions that the g-type lysozyme gene is expressed predominantly in tissues of organs exposed to the external environment or in hematopoietic tissues (Hikima et al., 2001; Zheng et al., 2007). The expression pattern of g-type lysozyme genes has been investigated in various organisms. In chickens, a restricted expression pattern of the g-type lysozyme was found; however, it was only expressed in the bone marrow and lungs, and not in the oviduct, providing an explanation as to why its absence was observed in chicken egg white (Nakano and Graf, 1991). In contrast, g-type lysozyme was quite abundant in the egg white of many other birds, including geese, ostriches and swans (Irwin and Gong, 2003). Nile et al. (2004) identified a second chicken g-type lysozyme sequence (chicken g2) that was expressed in the liver, kidneys and intestines. In humans, two g-type lysozymes were identified, but neither of these genes was widely expressed in fetal and adult tissues (Irwin and Gong, 2003). These restricted distribution patterns in birds and mammals contrasted 


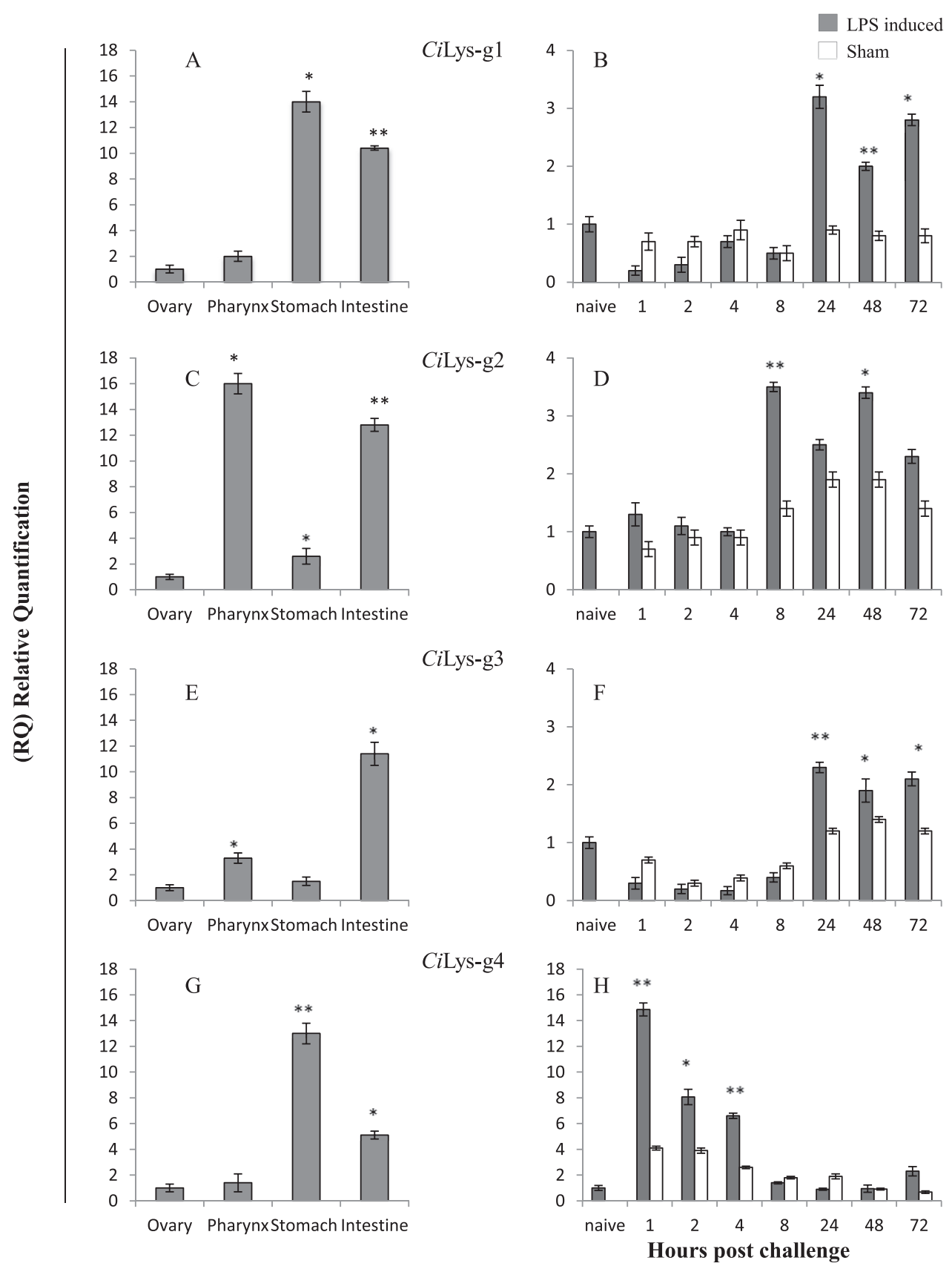

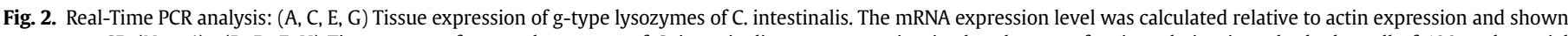

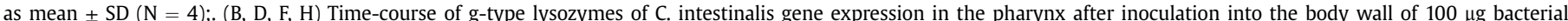

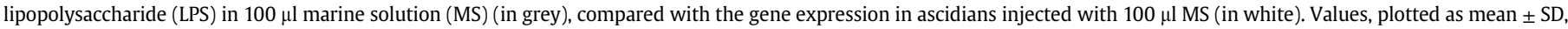

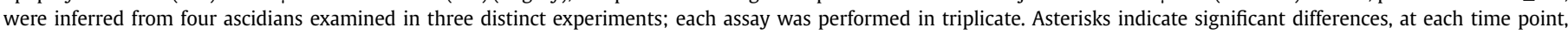
between LPS and MS inoculation (post hoc Tukey's $t$-test). ${ }^{*} \mathrm{P}<0.05$; ${ }^{* *} \mathrm{P}<0.01$.

with the broad expression patterns of g-type lysozymes in fish and invertebrate species. G-type lysozymes have been detected in the spleen, kidneys, gills, skin, heart, intestines and blood of Japanese flounder, orange-spotted grouper, large yellow croaker, Atlantic cod and grass carp (Hikima et al., 2001; Savan et al., 2003; Yin et al., 2003; Zheng et al., 2007), as well as in the gills, mantle, hepatopancreas, hemocytes and muscles in Mytilus galloprovincialis (Wang et al., 2012). Real-Time analysis has shown that C. intestinalis g-type lysozymes are upregulated in the pharynx after LPS challenge, in particular, CiLys-g1, CiLys-g2 and CiLys-g3 gene expression were significantly boosted at 24-72 h, while CiLys-g4 gene expression was significantly boosted at $1 \mathrm{~h}$ and $2-4 \mathrm{~h}$ and decreased at 8-48 h, supporting a defensive role for CiLys-g lysozymes. All of these findings suggest a functional role for CiLys-g(14 ) in innate immune defense and in the intracellular digestion of bacteria in $C$. intestinalis, a marine invertebrate that feeds by filtering seawater and which is often exposed to high concentrations of microorganisms.

\section{Acknowledgements}

This work was supported by a research grant from the Italian Ministry of Education (PRIN 2006 and 2010-2011 n. 20109XZEPR_007), co-funded by the University of Palermo. We 
thank M. Guarcello for collecting ascidians for the maintenance of the aquaria.

\section{Appendix A. Supplementary data}

\section{Supplementary data related to this article can be found at http://} dx.doi.org/10.1016/j.dci.2016.09.010.

\section{References}

Banks, J.G., Board, R.G., Sparks, N.H., 1986. Natural antimicrobial systems and their potential in food preservation of the future. Biotechnol. Appl. Biochem. 8 103-147.

Beintema, J.J., Terwisscha van Scheltinga, A.C., 1996. Plant lysozymes. In: Jollès, P. (Ed.), Lysozymes: Model Enzymes in Biochemistry and Biology. Birkhäuser Verlag, Basel, pp. 75-86.

Bonura, A, Vizzini, A., Salerno, G., Parrinello, N., Longo, V., Colombo, P. 2009. Isolation and expression of a novel MBL-like collectin cDNA enhanced by LPS injection in the body wall of the ascidian Ciona intestinalis. Mol. Immunol. 46 2389-2394.

Canfield, R.E., McMurry, S., 1967. Purification and characterization of a lysozyme from goose egg white. Biochem. Biophys. Res. Commun. 26, 38-42.

Delsuc, F., Brinkmann, H., Chourrout, D., Philippe, H., 2006. Tunicates and not cephalochordates are the closest living relatives of vertebrates. Nature 439 965-968.

Fastrez, J., 1996. Phage lysozymes. In: Jollès, P. (Ed.), Lysozymes: Model Enzymes in Biochemistry and Biology. Birkhäuser Verlag, Basel, pp. 35-64.

Ferrari, R., Callerio, C., Podio, G., 1959. Antiviral activity of lysozyme. Nature 183, 548.

Fu, G.H., Bai, Z.Y., Xia, J.H., Liu, F., Liu, P., Yue, G.H., 2013. Analysis of two lysozyme genes and antimicrobial functions of their recombinant proteins in Asian seabass. PLoS One 8 (11), e79743.

Grinde, B., 1989. Lysozyme from rainbow trout, Salmo gairdneri Richardson, as an antibacterial agent against fish pathogens. J. Fish. Dis. 12, 95-104.

Hancock, R.E., Scott, M.G., 2000. The role of antimicrobial peptides in animal defenses. Proc. Natl. Acad. Sci. U. S. A. 97, 8856-8861.

Hikima, J., Minagawa, S., Hirono, I., Aoki, T., 2001. Molecular cloning, expression and evolution of the Japanese flounder goose-type lysozyme gene, and the lytic activity of its recombinant protein. Biochim. Biophys. Acta 30, 35-44.

Holtje, J.V., 1996. Bacterial lysozymes. In: Jollès, P. (Ed.), Lysozymes: Model Enzymes in Biochemistry and Biology. Birkhäuser Verlag, Basel, pp. 65-74.

Hultmark, D., 1996. Insect lysozymes. In: Jollès, P. (Ed.), Lysozymes: Model Enzymes in Biochemistry and Biology. Birkhäuser Verlag, Basel, pp. 87-102.

Ibrahim, H.R., Aoki, T., Pellegrini, A., 2002. Strategies for new antimicrobial proteins and peptides: lysozyme and aprotinin as model molecules. Curr. Pharm. Des. 8 $671-693$.

Irwin, D.M., Gong, Z.Y., 2003. Molecular evolution of vertebrate goose-type lysozyme genes. J. Mol. Evol. 56, 234-242.

Jollès, J., Jollès, P., 1975. The lysozyme from Asterias rubens. Eur. J. Biochem. 54, $19-23$

Jollès, P., Jollès, J., 1984. What's new in lysozyme research? Always a model system, today as yesterday. Mol. Cell Biochem. 63, 165-189.

Kyomuhendo, P., Myrnes, B., Nilsen, I.W., 2007. A cold-active salmon goose-type lysozyme with high heat tolerance. Cell Mol. Life Sci. 64, 2841-2847.

Lee-Huang, S., Huang, P.L., Sun, Y., 1999. Lysozyme and RNases as antiHIVcomponents in beta-core preparations of human chorionic gonadotropin. Proc. Natl. Acad. Sci. U. S. A. 96, 2678-2681.

Mohanty, B.R., Sahoo, P.K., 2010. Immune responses and expression profiles of some immune-related genes in Indian major carp, Labeo rohita to Edwardsiella tarda infection. Fish Shelliish Immunol. 28, 613-621.

Nakano, T., Graf, T., 1991. Goose-type lysozyme gene of the chicken: sequence genomic organization and expression reveals major differences to chicken type lysozyme gene. Biochim. Biophys. Acta 1090, 273-276.

Nile, C.J., Townes, C.L., Michailidis, G., Hirst, B.H., Hall, J., 2004. Identification of chicken lysozyme g2 and its expression in the intestine. Cell Mol. Life Sci. 1 $2760-2766$.

Nilsen, I.W., Myrnes, B., Edvardsen, R.B., Chourrout, D., 2003. Urochordates carry multiple genes for goose-type lysozyme and no genes for chicken- o invertebrate-type lysozymes. Cell. Mol. Life Sci. 60, 2210-2218.

Nilsen, IW Øverbø, K., Sandsdalen, E. Sandaker, E., Sletten, K., Myrnes, B., 1999. Protein purification and gene isolation of chlamysin, a cold-active lysozyme-like enzyme with antibacterial activity. FEBS Lett. 464, 153-158.

Ogundele, M.O., 1998. A novel anti-inflammatory activity of lysozyme: modulation of serum complement activation. Mediat. Inflamm. 7, 363-365.

Parrinello, N., Vizzini, A., Arizza, V., Salerno, G., Parrinello, D., Cammarata, M. Giaramita, F.T., Vazzana, M., 2008. Enhanced expression of a cloned and sequenced Ciona intestinalis TNF alpha like (CiTNF alpha) gene during the LPS induced inflammatory response. Cell Tissue Res. 334, 305-317.

Parrinello, N., Vizzini, A., Salerno, G., Sanfratello, M.A., Cammarata, M., Arizza, V., Vazzana, M., Parrinello, D., 2010. Inflamed adult pharynx tissues and swimming larva of Ciona intestinalis share CiTNFalpha-producing cells. Cell Tissue Res. 341, $299-311$.

Prager, E.M., Jollès, P., 1996. Animal lysozymes c and g: an overview. In: Jollès, P. (Ed.), Lysozymes: Model Enzymes in Biochemistry and Biology. Birkhäuser Verlag, Basel, pp. 9-31.

Prager, E.M., Wilson, A.C., Arnheim, N., 1974. Widespread distribution of lysozyme g in egg white of birds. J. Biol. Chem. 249, 7295-7297.

Proctor, V.A., Cunningham, F.E., 1988. The chemistry of lysozyme and its use as a food preservative and a pharmaceutical. Crit. Rev. Food Sci. Nutr. 26, 359-395.

Rymuszka, A., Studnicka, M., Siwicki, A.K., Sierosławska, A., Bownik, A., 2005. The immunomodulatory effects of the dimer of lysozyme (KLP-602) in carp (Cyprinus carpio L) -in vivo study. Ecotoxicol. Environ. Saf. 61, 121-127.

Samarayanake, Y.H., Samaranayake, L.P., Wu, P.C., So, M., 1997. The antifungal effect of lactoferrin and lysozyme on Candida krusei and Candida albicans. APMIS 105, 875-883.

Sava, G. Benetti, A., Ceschia, V., Pacor, S., 1989. Lysozyme and cancer: role of exogenous lysozyme as anticancer agent (review). Anticancer Res. 9, 583-591.

Savan, R., Aman, A., Sakai, M., 2003. Molecular cloning of G-type lysozyme cDNA in common carp (Cyprinus carpio L.). Fish Shellfish Immunol. 15, 263-268.

Simpson, R.J., Morgan, F.J., 1983. Isolation and complete amino acid sequence of a basic low molecular weight protein from black swan egg white. Int. J. Pept. Protein Res. 22, 476-481.

Smirnow, J., Wislowska, M., 2001. Lysozyme and its biological value in rheumatoidarthritis (RA). Arthritis Res. 3 (Suppl. A), P014.

Thunnissen, A.M., Rozeboom, H.J., Kalk, K.H., Dijkstra, B.W., 1995. Structure of the 70-kDa soluble lytic transglycosylase complexed with bulgecin A. Implications for the enzymatic mechanism. Biochemistry 34, 12729-12737.

Tsagkogeorga, G., Turon, X., Hopcroft, R.R., Tilak, M.K., Feldstein, T., Shenkar, N., Loya, Y., Huchon, D., Douzery, E.J., Delsuc, F., 2009. An updated 18S rRNA phylogeny of tunicates based on mixture and secondary structure models. BMC Evol. Biol. 5, 9-187.

Valisena, S., Varaldo, P.E., Ingianni, A., Fontana, R., 1996. Modulatory effects of henegg-white lysozyme on immune response in mice. New Microbiol. 19, $15-24$.

Vizzini, A., Di Falco, F., Parrinello, D., Sanfratello, M.A., Cammarata, M., 2016. Transforming growth factor $\beta$ (CiTGF- $\beta$ ) gene expression is induced in the inflammatory reaction of Ciona intestinalis. Dev. Comp. Immunol. 55, $102-110$.

Vizzini, A., Falco, F.D., Parrinello, D., Sanfratello, M.A., Mazzarella, C., Parrinello, N., Cammarata, M., 2015a. Ciona intestinalis interleukin 17-like genes expression is upregulated by LPS challenge. Dev. Comp. Immunol. 48, 129-137.

Vizzini, A., Parrinello, D., Sanfratello, M.A., Mangano, V., Parrinello, N., Cammarata, M., 2013. Ciona intestinalis peroxinectin is a novel component of the peroxidase-cyclooxygenase gene superfamily upregulated by LPS. Dev. Comp. Immunol. 41, 59-67.

Vizzini, A., Parrinello, D., Sanfratello, M.A., Salerno, G., Cammarata, M., Parrinello, N., 2012. Inducible galectins are expressed in the inflamed pharynx of the ascidian Ciona intestinalis. Fish Shellfish Immunol. 32, 101-109.

Vizzini, A., Parrinello, D., Sanfratello, M.A., Trapani, M.R., Mangano, V., Parrinello, N., Cammarata, M., 2015b. Upregulated transcription of phenoloxidase genes in the pharynx and endostyle of Ciona intestinalis in response to LPS. J. Invertebr. Pathol. 126C, 6-11.

Wang, Q., Zhang, L., Zhao, J., You, L., Wu, H., 2012. Two goose-type lysozymes in Mytilus galloprovincialis: possible function diversification and adaptive evolution. PLoS One 7 (9), e45148.

Yin, Z.X., He, J.G., Deng, W.X., Chan, S.M., 2003. Molecular cloning, expression of orange spotted grouper goose-type lysozyme cDNA, and lytic activity of its recombinant protein. Dis. Aquat. Org. 55, 117-123.

Zeng, L., Swalla, B.J., 2005. Molecular phylogeny of the protochordates: chordate evolution. Can. J. Zool. 83, 24-33.

Zhang, S.H., Zhu, D.D., Chang, M.X., Zhao, Q.P., Jiao, R., Huang, B., Fu, J.P., Liu, Z.X. Nie, P., 2012. Three goose-type lysozymes in the gastropod Oncomelania hupensis: cDNA sequences and lytic activity of recombinant proteins. Dev. Comp. Immunol. 36, 241-246.

Zhang, X., Wu, F.X., Sun, M., Sang, O.Y., Wang, Y.J., Wang, X.K., 2008. Study on antimicrobial and antiviral activities of lysozyme from marine strain S-12-86 in vitro. Agric. Sci. China 7, 112-116.

Zhao, J.M., Song, L.S., Li, C.H., Zou, H.B., Ni, D.J., Wang, W., et al., 2007. Molecular cloning of an invertebrate goose-type lysozyme gene from Chlamys farreri, and lytic activity of the recombinant protein. Mol. Immunol. 44, 1198-1208.

Zheng, W., Tian, C., Chen, X., 2007. Molecular characterization of goose-type lysozyme homologue of large yellow croaker and its involvement in immune response induced by trivalent bacterial vaccine as an acute-phase protein. Immunol. Lett. 113, 107-116. 